\title{
Deubiquitinating enzyme USP33 restrains docetaxel-induced apoptosis via stabilising the phosphatase DUSP1 in prostate cancer
}

\author{
Fei Guo ${ }^{1} \cdot$ Chao Zhang $^{1} \cdot$ Fubo Wang ${ }^{1} \cdot$ Wei Zhang ${ }^{1} \cdot$ Xiaolei Shi ${ }^{1}$ Yasheng Zhu ${ }^{1} \cdot$ Ziyu Fang $^{1} \cdot$ Bo Yang ${ }^{1}$ • \\ Yinghao Sun ${ }^{1}$
}

Received: 17 June 2019 / Revised: 27 November 2019 / Accepted: 28 November 2019 / Published online: 19 December 2019

(c) The Author(s), under exclusive licence to ADMC Associazione Differenziamento e Morte Cellulare 2019

\begin{abstract}
The treatment of castration-resistant prostate cancer (CRPC) still faces many challenges. Docetaxel is a chemotherapeutic drug commonly used in CRPC patients. However, docetaxel-based chemotherapy usually causes docetaxel resistance, partially due to the resistance of CRPC cells to docetaxel-induced apoptosis. Here, we report that the deubiquitinating enzyme ubiquitin-specific protease 33 (USP33) inhibits docetaxel-induced apoptosis of prostate cancer cells, including androgenindependent prostate cancer cells. USP33 is overexpressed in prostate cancer cells and tissues. We found that knockdown or knockout of USP33 enhanced docetaxel-induced apoptosis of prostate cancer cells, accompanied by increased phosphorylation of the cJUN NH2-terminal kinase (JNK). After blocking docetaxel-induced JNK activation using the JNK inhibitor SP600125 or siRNA targeting JNK, the USP33 knockout-enhanced apoptosis was reversed. Furthermore, we found that USP33 could interact with the phosphatase DUSP1 to negatively regulate the activation of JNK, while USP33 knockdown promoted the proteasomal degradation of DUSP1. Mechanistically, we found that USP33 could inhibit the Lys48 (K48)-linked polyubiquitination of DUSP1. More importantly, DUSP1 overexpression could reverse the USP33 knockdowninduced JNK activation and apoptosis in docetaxel-treated prostate cancer cells. Therefore, USP33 overexpression in prostate cancer may contribute to docetaxel resistance by inhibiting the degradation of its partner DUSP1, leading to impaired JNK activation and apoptosis. Our study suggests that USP33-DUSP1-JNK may be a key signalling module mediating the docetaxel resistance of CRPC, indicating that USP33 is a potential novel therapeutic target in CRPC.
\end{abstract}

\section{Introduction}

Prostate cancer is one of the most common malignant tumours in developed countries, and its incidence in China is gradually increasing $[1,2]$. The treatment of prostate

These authors contributed equally: Fei Guo, Chao Zhang, Fubo Wang

Edited by V. D'Angiolella

Supplementary information The online version of this article (https:// doi.org/10.1038/s41418-019-0473-8) contains supplementary material, which is available to authorised users.

Bo Yang

yangbochanghai@126.com

Yinghao Sun

sunyhsmmu@126.com

1 Department of Urology, Changhai Hospital, The Second Military Medical University, 200433 Shanghai, China cancer, especially castration-resistant prostate cancer (CRPC), is a global challenge [3]. Docetaxel is a microtubule-targeting chemotherapeutic agent. It causes microtubule damage, which induces mitotic arrest in proliferating cells and activates the intrinsic or mitochondrial apoptosis pathway [4]. Furthermore, it is believed that docetaxel promotes cancer cell apoptosis mainly through JNK activation [5]. A docetaxel-based regimen is a commonly used cytotoxic therapeutic for CRPC patients. Unfortunately, patients usually develop docetaxel resistance after docetaxel treatments partly due to the inhibition of JNK apoptotic signalling [5]. Therefore, it is necessary to further elucidate the mechanisms involved in docetaxelinduced JNK activation and apoptosis.

As one of the most important post-translational modifications, ubiquitin (Ub) modification plays an important role in tumourigenesis and tumour progression [6]. Ub modification is a reversible process, which is mainly countered by deubiquitinases. Deubiquitinases can remove $\mathrm{Ub}$ chain(s) from protein substrates and thus regulate the 
degradation, localisation and activation of the substrates [7]. In recent years, several deubiquitinating enzymes have been found to play an important role in tumour cells, and tests of a variety of small-molecule compounds targeting deubiquitinases suggest that deubiquitinases have become emerging targets for anticancer therapeutics [8, 9].

USP33 (ubiquitin-specific protease 33), also known as VDU1 (VHL-interacting deubiquitinating enzyme 1), is a deubiquitinating enzyme [10]. It has been reported that USP33 promotes the replication of centrosomes during the $\mathrm{S}$ and $\mathrm{G} 2 / \mathrm{M}$ phases of the cell cycle by mediating the deubiquitination of the CP110 protein [11]. In addition, USP33 also promotes Slit signalling by binding to the intracellular portion of ROBO1, thus inhibiting breast cancer cell migration [12]. USP33 has been implicated in the pathogenesis of many other cancers, such as colorectal cancer and lung cancer [13-15]. Both in androgenstimulated prostate cancer cells and in tumour tissues of CRPC tumour-bearing mice receiving androgen deprivation therapy, the expression of USP33 is increased [16, 17]. However, the role of USP33 in prostate cancer, especially CRPC, has not been clearly elucidated.

DUSP1 (dual-specificity phosphatase-1), also known as MKP-1 (MAP kinase phosphatase 1), is a phosphatase primarily suggested to negatively regulate the activation of JNKs, extracellular signal-regulated kinases (ERKs), and p38 MAPK [18]. DUSP1 is highly expressed in prostate cancer and is negatively correlated with apoptosis of prostate cancer cells [19]. In other tumours, such as non-small cell lung cancer, pancreatic cancer, and breast cancer, DUSP1 can affect cisplatin-, gemcitabine- or paclitaxelinduced tumour cell apoptosis by modulating the phosphorylation of JNK and p38 MAPK [20-22]. However, the post-translational modification and regulation of DUSP1 itself in tumour cells, especially in prostate cancer cells, has not been completely understood.

Here, we report that USP33 is overexpressed in prostate cancer cells and tissues. We found that USP33 overexpression in prostate cancer cells, including androgenindependent prostate cancer cells, may contribute to impaired JNK activation and apoptosis after docetaxel treatments, possibly by removing Ub chains from Lys48 (K48) Ub-linked DUSP1. Our study provides mechanistic insights into the docetaxel resistance of CRPC and indicates USP33 as a potential novel target for CRPC treatments.

\section{Materials and methods}

\section{Cell culture}

The prostate cancer cell lines (LNCaP, ATCC number: CRL-1740; C4-2B, ATCC number: CRL-3315; and PC3,
ATCC number: CRL-1435) were maintained in RPMI medium 1640 (Gibco, 22400-089, Grand Island, NY, USA) supplemented with $10 \%$ fetal bovine serum (FBS) (Gibco, $10099141 \mathrm{C}), 2 \mathrm{mM}$ L-glutamine and $25 \mathrm{mM}$ HEPES. The normal prostate epithelial cell line (RWPE-1; ATCC number: CRL-11609) was cultured in keratinocyte serum-free medium (Gibco, 17005-042). HEK-293 cells (ATCC number: CRL-1573) were cultured in DMEM (Gibco, 11965-092) supplemented with 10\% FBS. The prostate cancer cells used in our study had been recently authenticated by Biowing Biotechnology Co., Ltd. (Shanghai, China) and Integrated Biotech Solutions Co., Ltd. (Shanghai, China) by using short tandem repeat (STR) analysis as described in 2012 in an ANSI Standard (ASN-0002) by the ATCC Standards Development Organization (SDO). Fluorescent quantitative PCR (qRT-PCR) and primers for mycoplasma (Forward: GGGAGCAAACAGGATTAGAT ACCCT and Reverse: TGCACCATCTGTCACTCTGTT AACCTC) were used to determine whether cells had mycoplasma contamination.

\section{Mice, reagents and antibodies}

Six- to 8-week-old male BALB/c nude mice were purchased from Joint Ventures Sipper BK Experimental Animal (Shanghai, China). All animal experiments were performed in accordance with the National Institutes of Health Guide for the Care and Use of Laboratory Animals and with the approval of the Scientific Investigation Board of Second Military Medical University, Shanghai. Antibodies specific to HA-tag (HA.C5, ab18181), Myc-tag (Myc.A7, ab18185), Flag-tag (M2, ab49763), $\beta$-actin (AC15, ab6276) and DUSP1 (ab195261) were obtained from Abcam Inc. (Cambridge, MA, USA). Antibodies specific for IkBa (44D4, \#4812), phospho-ERK1/2 (Thr202/Tyr204) (197G2, \#4377), JNK (\#9252), phospho-JNK1/2 (Thr183/ Tyr185) (81E11, \#4668), p38 MAPK (D13E1, \#8690), phospho-p38 (Thr180/Tyr182) (12F8, \#4631), phosphoAKT (Thr308, \#9275), cleaved caspase 3 (Asp175) (5A1E, \#9664), cleaved PARP (Asp214) (D64E10, \#5625), ubiquitin (P4D1, \#3936), K48-linkage specific polyubiquitin (\#4289) and Myc-tag (9B11, Sepharose bead conjugate, \#3400) were purchased from Cell Signaling Technology (Beverly, MA, USA). Anti-USP33 antibody (HPA005719) was purchased from Sigma Aldrich (St. Louis, MO, USA). Pierce $^{\mathrm{TM}}$ Protein A/G Agarose (20422) was obtained from ThermoFisher Scientific (Rockford, IL, USA). The inhibitors SP600125 (HY-12041) and SB203580 (HY-10256) were obtained from MedChemExpress (Monmouth, NJ). MG132 (Cat. No. S2619) and docetaxel (Cat. No. S1148) were obtained from Selleck Chemicals (Houston, TX, USA). Other non-specified reagents were from Sigma Aldrich (St. Louis, MO, USA). 


\section{Sequences, plasmids, transfection and RNA interference}

Flag- or Myc-tagged vectors of USP33 (GenBank No. NM_015017.4), DUSP1 (GenBank No. NM_004417.3) and the indicated mutants were subcloned into the pcDNA3.1 vector as described [23]. HA-Ub and the mutant vectors were gifts from Prof W.L. Chen (Shenzhen University, Shenzhen, China). For transient transfection of plasmids in prostate cancer cells, the X-tremeGENE HP reagents were used according to the manufacturer's instructions (Cat. No. 06366546 001, Roche, Welwyn Garden City, UK). For transient knockdown of a specific molecule, $20 \mathrm{nM}$ siRNA duplexes specific for the molecule were transfected using INTERFERin ${ }^{\text {TM}}-$ HTS according to the standard protocol (Cat. No. 409-50, Polyplustransfection, Illkirch, France). A nonsense sequence was used as a control siRNA. The siRNA duplexes specific for DUSP1 (sc-35937), JNK1 (sc-29380) and JNK2 (sc-39101) were from Santa Cruz Biotechnology (Dallas, TX, USA). The siRNAs specific for USP33 were designed and synthesised by Shanghai GenePharma Co., Ltd. (Shanghai, China). Generally, these siRNAs were designed obey the same design rules as coding genes. The sequences are listed in Supplemental Table S1.

\section{RNA quantification}

Total RNA was purified by TRIzol (Thermo Scientific, Grand Island, NY, USA) from cells and reverse-transcribed using the ReverTra Ace qPCR RT Kit (Toyobo, Osaka, Japan). qRT-PCR was performed with diluted cDNA using SYBR Green PCR master mix (Toyobo, Osaka, Japan) and gene-specific primers on an ABI7500 instrument (Applied Biosystems, Foster City, CA, USA). The sequences of all primers are listed in Supplemental Table S1.

\section{Tissue arrays and immunohistochemistry}

Tissue arrays containing multiple human normal and cancerous prostate tissues were obtained from ServiceBio Co., Ltd. (Wuhan, China). Informed consent was obtained from all subjects. The tissue sections were immunostained following a standard protocol using anti-USP33 and antiDUSP1 antibodies. The EnVision ${ }^{+}$detection system (Dako) was used per the manufacturer's instructions. Immunostained tissue arrays were scored by multiplying the intensity $(0-3)$ and extent $(0-100)$ of staining for each tissue point, which was analysed by a pathologist at Changhai Hospital as described previously [24].

\section{CRISPR-Cas9-mediated depletion of USP33}

For the depletion of USP33, the pC3-U6-guide RNA-CMVRED (encoding guide RNA and red fluorescent protein) and
Cas9-IRES-EGFP (encoding Cas9 and green fluorescent protein) plasmids (kind gifts from Shanghai Biomodel Organism Science \& Technology Development Co., Shanghai, China) were cotransfected into PC3 cells. Three target sequences for guide RNA synthesis against USP33 were tested and one guide RNA sequence (5'TCGAAATCATTGTCCACATT- $3^{\prime}$ ) was the most efficient in depleting USP33. Cells with both red and green fluorescence were then sorted by using a Gallios flow cytometer (Beckman Coulter, Brea, CA). Sorted cells were cultured for 5-7 days, and clones propagated from single cells were selected. The depletion of USP33 was confirmed by both western blot and DNA sequencing.

\section{Immunoprecipitation and immunoblot}

Cell samples were lysed with IP lysis buffer (ThermoFisher Scientific) supplemented with a protease inhibitor cocktail (Abcam) for $15 \mathrm{~min}$ on ice and were then centrifuged at $12,000 \times g$ for $10 \mathrm{~min}$. The supernatants were collected, and the total protein concentrations were measured by using a BCA kit (Thermo Scientific). For immunoprecipitation, equal amounts of lysate were incubated with IgG, anti-USP33 or anti-DUSP1 plus protein $\mathrm{A} / \mathrm{G}$ agarose or anti-Myc Sepharose bead conjugates overnight at $4{ }^{\circ} \mathrm{C}$. Then, the beads were washed three times with IP lysis buffer and prepared for western blotting. The western blot assays were performed as described [23].

\section{Nanospray liquid chromatography-tandem mass spectrometry}

Immunoprecipitated USP33 complexes were separated on an SDS-PAGE gel as described previously [25]. After silver staining, each differential gel band was excised and then analysed by nano-ultra-performance liquid chromatography-electrospray ionisation tandem mass spectrometry as described [25].

\section{Polyubiquitination assay}

For analysis of ubiquitination of DUSP1, whole-cell extracts prepared with radioimmunoprecipitation assay buffer (50 mM Tris, $\mathrm{pH} 8.0,150 \mathrm{mM} \mathrm{NaCl}, 1 \%$ (vol/vol) Nonidet$\mathrm{P} 40,0.5 \%$ (wt/vol) sodium deoxycholate, $1 \%$ (wt/vol) SDS, and proteinase inhibitors) supplemented with $10 \mathrm{mM} \mathrm{N}$ ethylmaleimide were heat denatured in the presence of $1 \%$ SDS and then immunoprecipitated with the indicated antibodies and analysed by immunoblot as described [23].

\section{CCK8, BrdU incorporation and apoptosis assays}

The viability of LNCaP, C4-2B and PC3 cells was evaluated by a CCK8 assay (Dojindo, Japan) according to the 
manufacturer's instructions. The proliferation of $\mathrm{LNCaP}$, C4-2B and PC 3 cells was evaluated by a BrdU Cell Proliferation Assay Kit (Cell Signaling Technology, USA) according to the manufacturer's instructions. The Annexin V/PI apoptosis kit (BD, USA) was used to detect cell apoptosis according to the manufacturer's instructions.

\section{Xenograft model and in vivo treatment}

All animal experiments were conducted according to the National Institutes of Health Guide for the Care and Use of Laboratory Animals, with the approval of the Scientific Investigation Board of Second Military Medical University, Shanghai. PC3 cells $\left(1 \times 10^{7}\right)$ were subcutaneously injected into the back of the mice. For in vivo treatments, only inoculated mice $(n \geq 3)$ with established tumours of the required size $\left(70-100 \mathrm{~mm}^{3}, \sim 1\right.$ week after tumour cell inoculation) were selected. The remaining mice will be excluded from the analysis. A total of $20 \mu \mathrm{g}$ USP33 siRNA duplexes were incubated with in vivo-jetPEI reagents as suggested (Cat. No. 201-50G, Polyplus-transfection) and intratumourally administered. Docetaxel was intraperitoneally injected at $10 \mathrm{mg} / \mathrm{kg}$ once every 5 days for a total of three times from the time the tumour reached the required size $\left(70-100 \mathrm{~mm}^{3}\right)$ selected from all inoculated mice. All animal with established tumours of the required size and the same genotype were randomly assigned into four or two groups as needed. Technicians were blinded when measuring tumour sizes.

\section{Statistical analysis}

All statistical analyses were performed using GraphPad Prism 5.0 software. All experiments were repeated at least three times and $(n=3)$ for each experimental group. Statistical significance between the means of two groups was determined using Student's $t$ tests (normal distribution), Mann-Whitney $U$ tests (abnormal distribution) or Wilcoxon signed rank test (matched pairs). Statistical comparisons of the means of multiple groups were performed using oneway ANOVA or two-way ANOVA. Correlations between two variables were evaluated by using the Pearson correlation assay. Data are presented as the mean \pm s.d. $P<0.05$ was considered statistically significant.

\section{Results}

\section{USP33 is overexpressed in prostate cancer cells and tissues}

To explore the role of USP33 in prostate cancer, we first analysed the expression of USP33 in prostate cancer cell lines. We found that prostate cancer cell lines, especially CRPC cell lines (C4-2B and PC3), showed higher USP33 expression compared to the normal prostate cell line (RWPE-1) at both the mRNA and protein levels (Fig. 1a, b). Then, immunohistochemistry (IHC) staining of USP33 protein was performed in tissue microarrays containing both
Fig. 1 Overexpression of USP33 in prostate cancer. a, b The levels of USP33 were examined by Q-PCR (a) and western blot analysis (b) in four prostate cell lines. Data are shown as the mean \pm s.d. of triplicate samples derived from one representative experiment. Similar results were obtained in three independent experiments. $* * P<0.01 ; * * * P<0.001$. c Representative IHC images for USP33 protein in tissue microarrays. Typical staining images in the red box (left) were magnified and shown in adjacent rows (right). d IHC scores for USP33 staining in the indicated groups. Error bars represent the mean \pm s.d. $(n=24$, Wilcoxon signed rank test). ${ }^{* *} P<0.01$. a

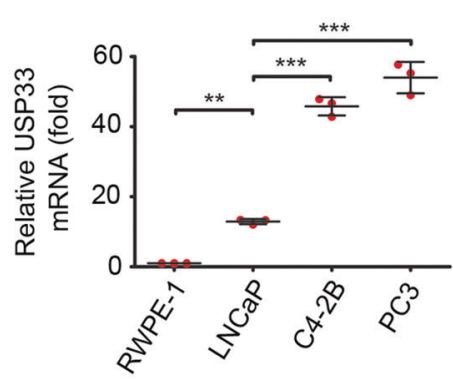

C

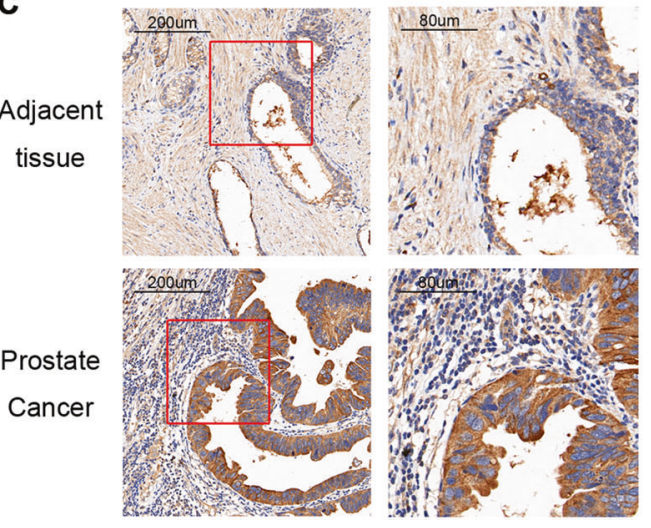

b

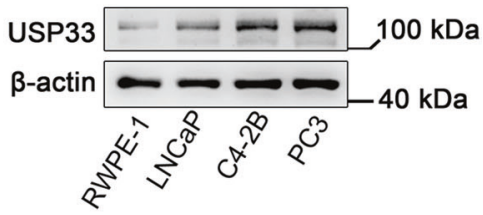

d

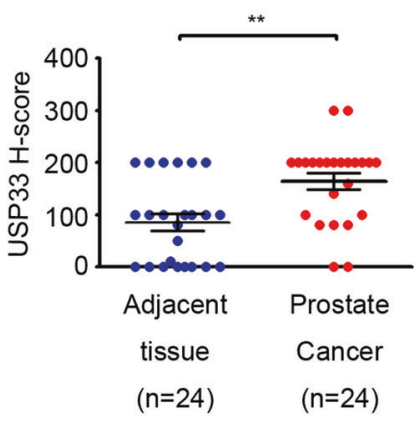


cancerous and matched adjacent normal tissues derived from prostate cancer patients. According to the EAU and AUA guidelines for prostate cancer treatment, radical surgical treatment for prostate cancer patients with distant metastasis or CRPC patients is not suggested [26, 27]. The specimens collected in this study were all specimens of localised prostate cancer after radical prostatectomy, and the prognosis was relatively good. Therefore, the survival data for these patients in our study are not available at present. The IHC results (Fig. 1c, d) showed that a higher level of USP33 protein was detected in human prostate cancer tissues than in matched adjacent normal tissues. These data suggest that USP33 is overexpressed in prostate cancer tissues and androgen-independent prostate cancer cells, indicating that USP33 may play a role in the development or progression of prostate cancer.

\section{USP33 inhibits apoptosis and enhances the viability of prostate cancer cells}

The upregulation of USP33 in prostate cancer raises the possibility that USP33 overexpression may be an important tumour-promoting factor in prostate cancer. To verify this hypothesis, we first tested siRNAs targeting USP33 for functional effects in prostate cancer cells. We transfected three different siRNAs targeting USP33 into the LNCaP, C4-2B and PC3 prostate cancer cell lines. All of the siRNAs for USP33 (\#1 to \#3) could suppress the expression of USP33 protein in these cells (Supplementary Fig. S1A). Then, we tested cell viability after knocking down USP33 in the $\mathrm{LNCaP}, \mathrm{C} 4-2 \mathrm{~B}$ and $\mathrm{PC} 3$ prostate cancer cell lines. The CCK8 assay showed that USP33 was positively correlated with the viability of the three prostate cancer cell lines (Fig. 2a). Since the \#1 and \#2 USP33 siRNAs showed stronger suppression of USP33 protein levels, we used them for further analysis (Supplementary Fig. S1A). To exclude the potential off-target effects of USP33 knockdown, we transiently overexpressed two mutants of USP33 resistant to the \#1 and \#2 siRNAs (si-res) in USP33-silenced PC3 cells and examined cell viability. We found that these USP33 mutants could rescue the effects of USP33 knockdown on PC3 cells (Fig. 2b, Supplementary Fig. S1B, C), indicating that these two siRNA duplexes are suitable for subsequent experiments. To further investigate the function of USP33 in prostate cancer, we performed a BrdU incorporation experiment (an indicator of cell proliferation). The results of BrdU incorporation demonstrated that USP33 knockdown did not significantly affect the proliferation of prostate cancer cells (Fig. 2c).

Apoptosis plays a critical role in affecting cell viability in response to anticancer therapies. Therefore, we tested the regulatory effect of USP33 on the apoptosis of androgenindependent prostate cancer cells. We found that knockdown of USP33 could promote the apoptosis of prostate cancer cells under normal conditions slightly and significantly after treatment with docetaxel (Fig. 2d, Supplementary Fig. S2). We also found that more cleaved PARP1 and caspase 3 were detected in C4-2B and PC3 cells with or without docetaxel treatments after USP33 knockdown (Fig. 2e).

We further validated the roles of USP33 in prostate cancer by depleting USP33 expression using the CRISPRCas9-mediated genome editing technique in PC3 cells. We randomly selected three clones of USP33 wild-type PC3 cells (USP33 $3^{+/+}, \# 1-\# 3$ ) and three clones of USP33 knockout PC3 cells (USP33 ${ }^{-1-}$, \#1-\#3) for apoptosis analysis (Supplementary Fig. S3A). We found that all clones of $\mathrm{USP}^{-1-}$ cells are more susceptible to apoptosis than wildtype cells in the presence or absence of docetaxel (Supplementary Fig. S3B, C). This indicated that there is no significant clonal difference in the function of USP33 in PC3 cells. Therefore, we selected USP $33^{+/+}(\# 1)$ and USP $33^{-1-}$ (\#1) for subsequent research. Consistent with the results obtained by USP33 interference, USP $33^{-1-}$ PC3 cells showed decreased cell viability, and this effect could be rescued by transfection of wild-type (WT) USP33 (Fig. 3a). Therefore, the observed effects of USP33 in PC 3 cells should be attributed to USP33 knockout specifically and not to offtarget effects. Further, knockout of USP33 showed no effects on the proliferation of PC 3 cells (Fig. 3b) but made PC 3 cells more susceptible to apoptosis, especially after docetaxel treatment (Fig. 3c). It has been reported that mutations in specific cysteine and histidine sites of USP33 (C194S and H673Q in human USP33) could inactivate the enzymatic activity of USP33 [11, 28]. We found that WT-USP33 but not Mut-USP33 with a C194S/H673Q mutation (inactive deubiquitinating enzyme activity) could rescue PC3 cells from apoptosis (Fig. 3c, Supplementary Fig. S4). More cleaved PARP1 and caspase 3 were also detected in $\mathrm{USP}_{3}{ }^{-1-}$ PC 3 cells and Mut-USP33-transfected USP $33^{-1-}$ $\mathrm{PC} 3$ cells with or without docetaxel treatments compared to $\mathrm{USP}_{3} 3^{+/+} \mathrm{PC} 3$ cells and WT-USP33-transfected USP $33^{-/-}$ PC 3 cells (Fig. 3d). Our data suggest that USP33 can inhibit apoptosis and may thus contribute to docetaxel resistance in androgen-independent prostate cancer cells through its deubiquitinating enzyme activity.

\section{USP33 knockout promotes docetaxel-induced apoptosis by enhancing JNK activation}

To study the mechanism by which USP33 inhibited the apoptosis of prostate cancer cells, we analysed the activation of related signalling pathways in androgen-independent cells after USP33 knockdown. We found that the activation of JNK, but not p38 MAPK, ERK, AKT and NF-kB, was enhanced following USP33 knockdown in C4-2B and PC-3 

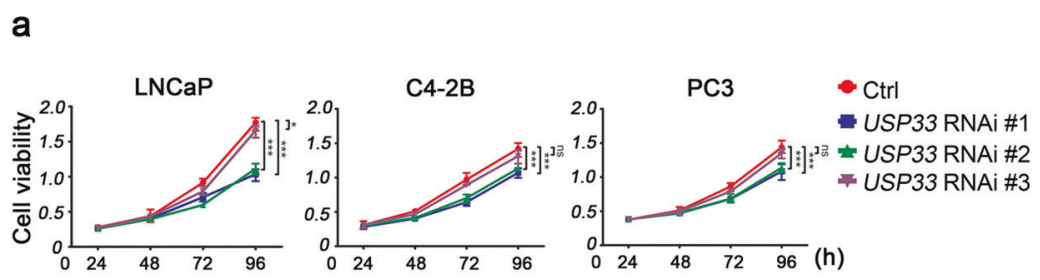

b

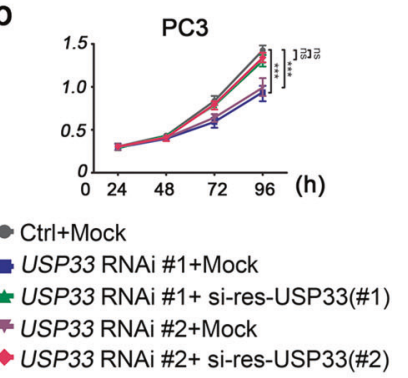

C

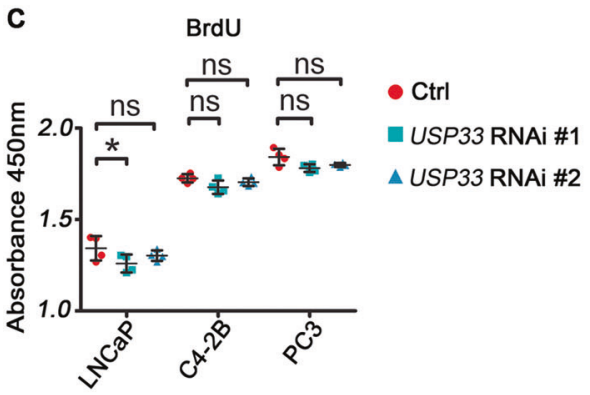

d

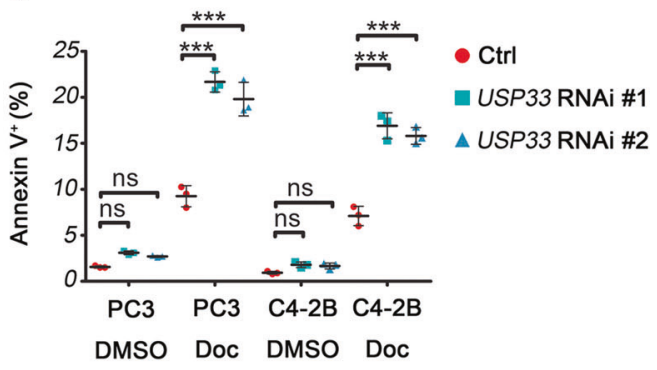

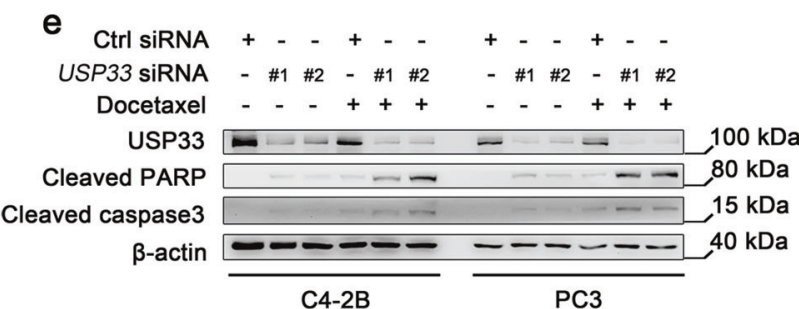

Fig. 2 USP33 knockdown promotes the apoptosis of prostate cancer cells. a Cell viability was measured by CCK8 assay at $24 \mathrm{~h}$, $48 \mathrm{~h}, 72 \mathrm{~h}$, and $96 \mathrm{~h}$ after knocking down USP33 (USP33 RNAi \#1 to $\# 3,20 \mathrm{nM}$ ) for $48 \mathrm{~h}$ in the indicated cells. b Cell viability was measured as in a after knocking down USP33 (USP33 RNAi \#1 and \#2, $20 \mathrm{nM}$ ) and transfecting PC3 cells with Ctrl vector (Mock), WTUSP33 (si-res \#1) (si-resistance \#1) or WT-USP33 (si-res \#2) vectors for $48 \mathrm{~h}$. c BrdU incorporation assay was performed in the cells transfected as in a at $24 \mathrm{~h}$. d PC3 and C4-2B cells were transfected with control (Ctrl) or USP33 siRNA (USP33 RNAi \#1 and \#2, $20 \mathrm{nM}$ ) for $48 \mathrm{~h}$ and then treated with DMSO or docetaxel (Doc) $(10 \mathrm{nM})$ for $48 \mathrm{~h}$ as indicated. Annexin $\mathrm{V}^{+}$apoptotic cells were quantified by

cells (Fig. 4a). After adding docetaxel, JNK activation was further significantly increased (Fig. 4a). We observed the same effect in USP $33^{-1-}$ PC3 cells. More importantly, WTUSP33 but not Mut-USP33 could rescue the activation of JNK (Fig. 4b). Therefore, the effects of USP33 on docetaxelinduced apoptosis in prostate cancer cells may require JNK signalling. When we added the JNK inhibitor SP600125 or transfected JNK1/2 siRNA into the cell culture system, the increase in cleaved caspase 3 and cleaved PARP caused by USP33 knockout was reversed (Fig. 4c). Moreover, the JNK inhibitor SP600125 or JNK1/2 siRNA could reverse the apoptosis of PC3 cells induced by docetaxel treatments after USP33 knockout (Fig. 4d). However, the addition of the p38 MAPK inhibitor SB203580 showed no obvious effect (Fig. 4c, d). Taken together, these data suggest that USP33
Annexin V/PI assay. Data and error bars in (a) to (d) represent the mean \pm s.d. of triplicate samples derived from one representative experiment. Similar results were obtained in three independent experiments. $* P<0.05$; $* * * P<0.001$; ns, not significant (one-way ANOVA followed by Bonferroni multiple comparison using PRISM software). e C4-2B and PC3 cells were transfected with control (Ctrl) or USP33 siRNA (USP33 RNAi \#1 and \#2) for $48 \mathrm{~h}$ and then treated with DMSO or docetaxel $(10 \mathrm{nM})$ for $24 \mathrm{~h}$ as indicated. The indicated molecules were examined by western blot. One representative experiment of three is shown. Similar results were obtained in three independent experiments.

impairs the apoptosis of androgen-independent prostate cancer cells by inhibiting JNK activation.

\section{USP33 interacts with DUSP1 in prostate cancer cells}

To further elucidate the underlying mechanism by which USP33 inhibited JNK activation, we performed liquid chromatography mass spectrometry (LC-MS) assays after immunoprecipitation (IP) of USP33 in PC3 cells. The MS data indicated that USP33 could interact with DUSP1 (Supplementary Fig. S5), a phosphatase that can dephosphorylate JNK, ERK or p38 MAPK separately under different conditions [18]. Then, we confirmed the endogenous interaction of USP33 and DUSP1 by performing IP with anti-USP33 antibody and found that USP33 interacted with 
a
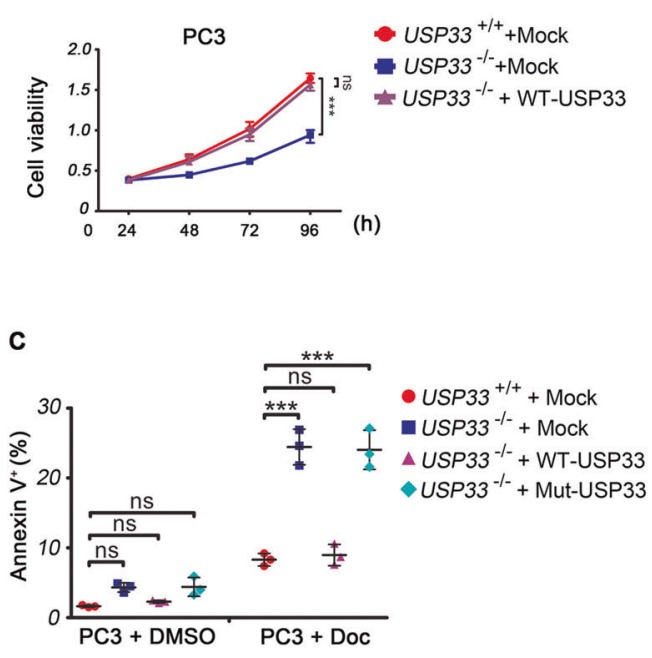

b
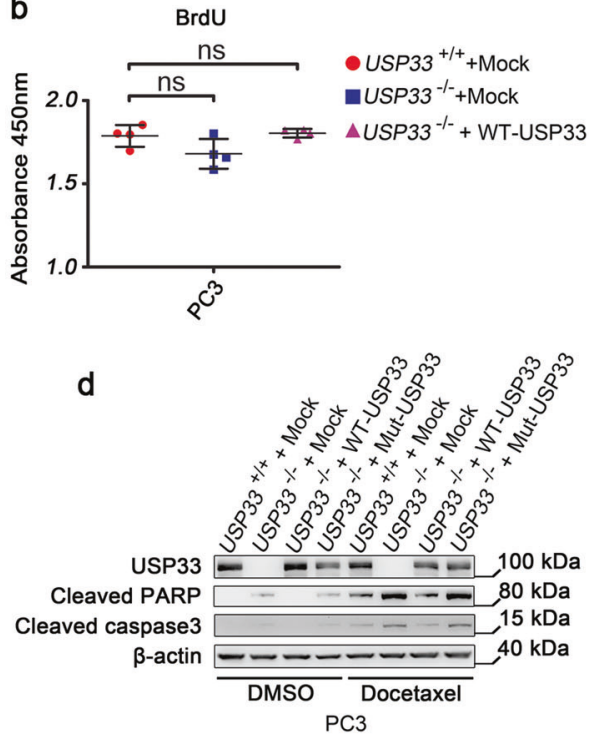

s.d. of triplicate samples derived from one representative experiment. Similar results were obtained in three independent experiments. $* * * P<0.001$; ns, not significant (one-way ANOVA followed by Bonferroni multiple comparison using PRISM software). d USP33 $3^{+/+}$ and USP $33^{-1-}$ PC 3 cells were transfected as in $\mathbf{c}$ and then treated with DMSO or docetaxel (Doc) $(10 \mathrm{nM})$ for $24 \mathrm{~h}$ as indicated. The indicated molecules were examined by western blot assay. One representative experiment of three is shown. Similar results were obtained in three independent experiments.

examined the effect of the proteasome inhibitor MG132 on the correlation between USP33 and DUSP1 expression levels. In the presence of the proteasome inhibitor MG132, the effects of USP33 on DUSP1 were rescued (Fig. 6a, b), indicating that USP33 inhibited the proteasomal degradation of DUSP1 in prostate cancer cells. Next, we examined the polyubiquitination of DUSP1 in C4-2B and PC-3 cells after USP33 knockdown. We found that DUSP1 could be polyubiquitinated in both cells, which was significantly increased in USP33-silenced cells (Fig. 6c).

To further elucidate the modification type of DUSP1 regulated by USP33, we cotransfected Myc-tagged DUSP1 and Flag-tagged USP33 with several HA-tagged Ub (wild type or mutant) into HEK293 cells and found that the wildtype $\mathrm{Ub}$ and the $\mathrm{Ub}$ with only intact Lys48 residue (mutate other Lys into Arg) (HA-Ub (K48)) could be removed from DUSP1 by USP33 (Supplementary Fig. S6). We confirmed the requirement of USP33 for removing K48 polyubiquitination of DUSP1 by using a K48 polyubiquitination specific antibody and found that USP33 knockdown significantly increased the K48-linked polyubiquitination of DUSP1 (Fig. 6d). Overexpression of the USP33 C194S/ H673Q mutant (Flag-USP33 mutant) in HEK293 cells could no longer dose-dependently decrease the level of K48-linked polyubiquitination of DUSP1 (Fig. 6e), indicating that the ubiquitin peptidase activity of USP33 was indispensable for 

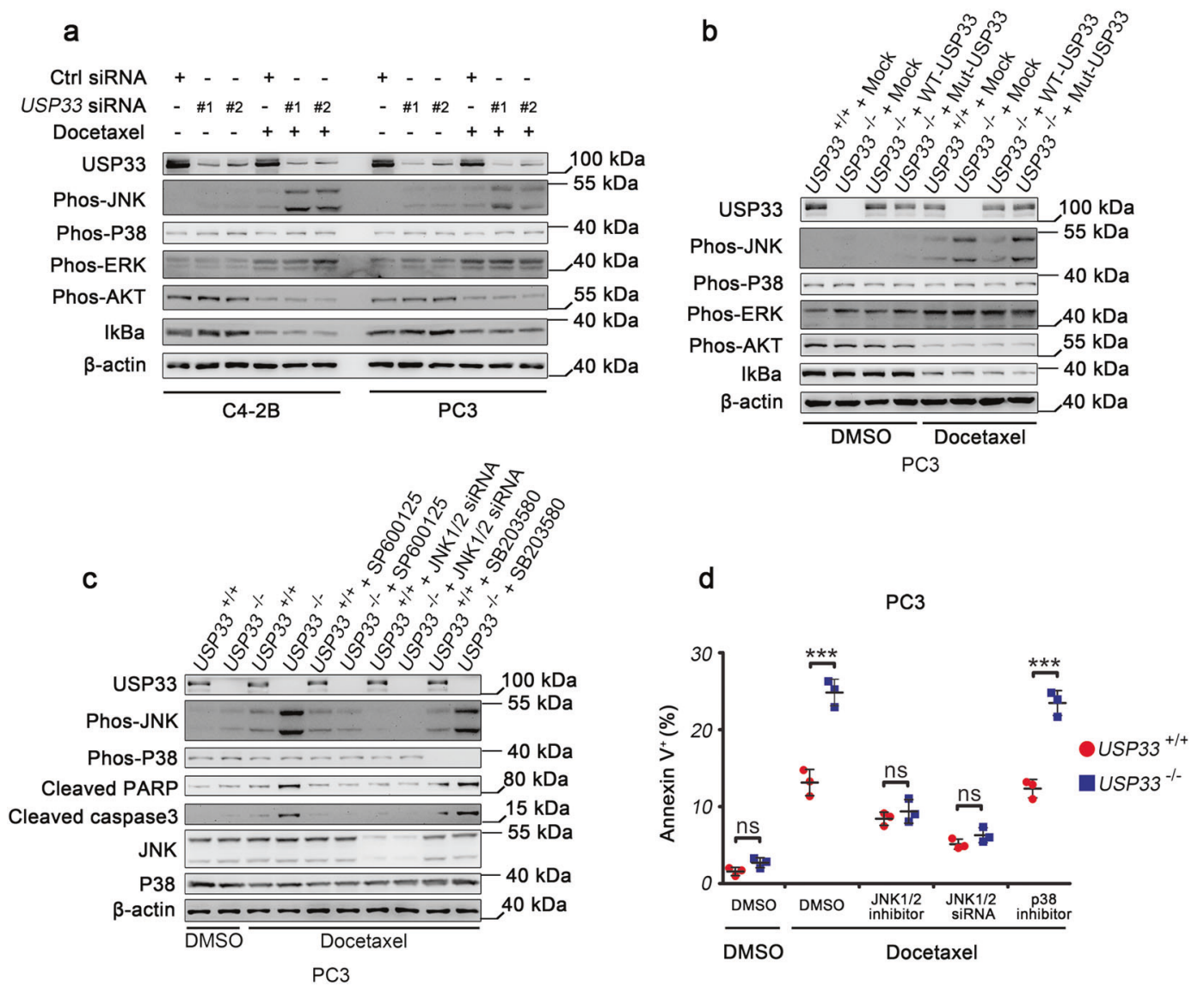

Fig. 4 JNK activation is required for USP33 knockout-mediated regulation of apoptosis in prostate cancer cells. a C4-2B and $\mathrm{PC} 3$ cells were transfected with control (Ctrl) or USP33 siRNA (USP33 RNAi \#1 and \#2, $20 \mathrm{nM}$ ) for $48 \mathrm{~h}$ and then treated with DMSO or docetaxel (Doc) $(10 \mathrm{nM})$ for $24 \mathrm{~h}$ as indicated. The indicated molecules were examined by western blot assay. b USP $33^{+/+}$and $\mathrm{USP}^{-1-}$ PC 3 cells were transfected with Mock, WT-USP33 or MutUSP33 vector as indicated for $48 \mathrm{~h}$ and then treated with DMSO or docetaxel (Doc) $(10 \mathrm{nM})$ for $24 \mathrm{~h}$ as indicated. The indicated molecules were examined by western blot assay. c USP $33^{+/+}$and $\mathrm{USP}^{-1-}$ PC3 cells were treated with DMSO or docetaxel (Doc) $(10 \mathrm{nM})$ for $24 \mathrm{~h}$ in the presence of SP600125 $(20 \mu \mathrm{M}), \mathrm{SB} 203580$

deubiquitinating K48 polyubiquitination of DUSP1. Subsequently, we verified in USP $33^{-1-}$ PC3 cells that the ubiquitination level of DUSP1 was significantly increased after knocking out USP33 and that the Flag-USP33 but not the Flag-USP33 mutant could reverse the K48-ubiquitination level of DUSP1 (Fig. 6f). Therefore, our data suggest that USP33 can bind and stabilise the DUSP1 protein by removing the K48 polyubiquitination chains from DUSP1.

\section{DUSP1 and USP33 synergistically inhibit docetaxel- induced activation of JNK and apoptosis of prostate cancer cell lines}

The above results suggest that USP33 inhibits the apoptosis of prostate cancer cells possibly by stabilising DUSP1 and

$(20 \mu \mathrm{M})$ or transfected with JNK1/2 siRNA $(20 \mathrm{nM})$ as indicated, and the indicated molecules were examined by western blot assay. One representative experiment of three is shown in a to c. Similar results were obtained in three independent experiments. d USP $33^{+/+}$and $\mathrm{USP}_{3}{ }^{-1-} \mathrm{PC} 3$ cells treated as in $\mathbf{c}$ were incubated with DMSO or docetaxel $(10 \mathrm{nM})$ for $48 \mathrm{~h}$. Annexin $\mathrm{V}^{+}$apoptotic cells were quantified by Annexin V/PI assay. Error bars represent the mean \pm s.d. of triplicate samples derived from one representative experiment. Similar results were obtained in three independent experiments. $* * * P<0.001$; ns, not significant (one-way ANOVA followed by Bonferroni multiple comparison using PRISM software).

inhibiting JNK activation. DUSP1 has been reported to enhance the chemoresistance of tumour cells by inhibiting the activation of JNK [22]. Therefore, we wondered whether the anti-apoptotic effect of USP33 depends on the dephosphorylation of JNK by stabilising DUSP1. We found that overexpression of DUSP1 could rescue PC3 cells from USP33 knockdown-induced apoptosis in the presence of docetaxel (Fig. 7a). In addition, overexpression of DUSP1 could also reverse the phosphorylation of JNK and the levels of cleaved PARP and caspase 3 induced by USP33 knockdown in PC3 cells treated with docetaxel (Fig. 7b). When we knocked down DUSP1 in PC3 cells, we found that the apoptosis of PC3 cells was promoted and was increased even further due to the synergistic effect of USP33 knockout (Fig. 7c). The phosphorylation of JNK 


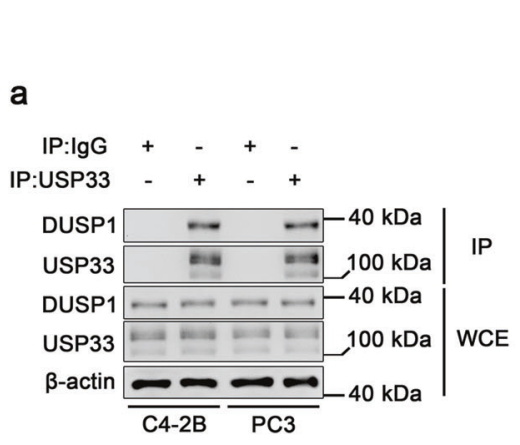

d

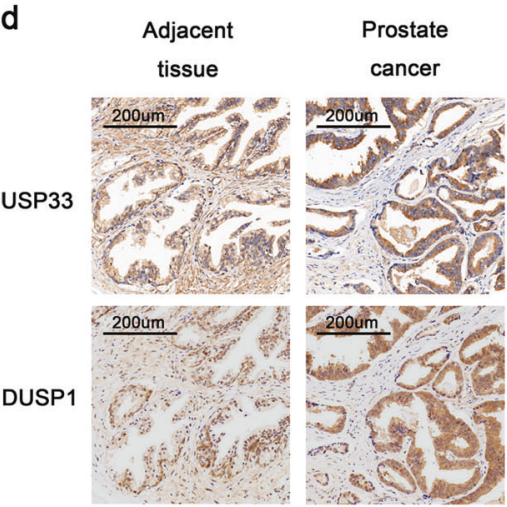

Fig. 5 USP33 interacts with DUSP1 and is positively correlated with DUSP1 levels. a C4-2B and PC3 cells were prepared for wholecell extracts (WCE), which were immunoprecipitated (IP) with antiUSP33 or IgG as indicated. The associated DUSP1 was examined by western blot. b PC3 cells transfected with Ctrl siRNA or USP33 siRNA (USP33 RNAi \#1), and USP33 ${ }^{+/+}$and USP33 ${ }^{-1-}$ PC3 cells were transfected with Mock, WT-USP33 or Mut-USP33 vector (siresistance (si-res) or not) as indicated for $48 \mathrm{~h}$. The indicated

and the cleavage of PARP and caspase 3 were also enhanced after knocking down DUSP1 (Fig. 7d). These data together validate that DUSP1 functions downstream of USP33 but upstream of JNK in the same signalling pathway and that USP33 inhibits the apoptosis of androgenindependent prostate cancer cells by stabilising DUSP1 and, in turn, inhibiting JNK activation.

\section{USP33 is a potential therapeutic target for prostate cancer}

To investigate the possible application of USP33 knockdown in tumour therapy, we tested the effects of USP33 siRNA in combination with docetaxel on established CRPC tumours. We found that repeated intratumoural injection of USP33 siRNAs could potentiate the effects of docetaxel in inhibiting tumour growth and decrease the protein level of DUSP1 in tumours (Fig. 8a-c). Moreover, USP33-deficient tumours were more sensitive to docetaxel treatment and showed lower protein levels of DUSP1 (Fig. 8d-f). These data indicate that USP33 knockdown may be used in the treatment of CRPC.

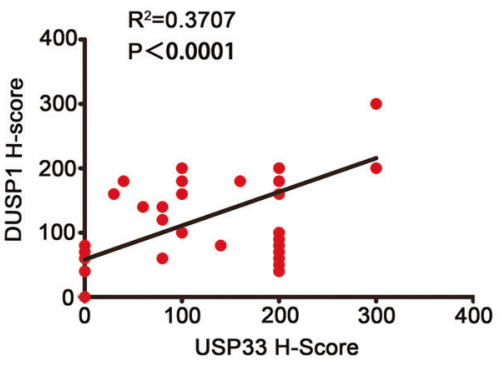

molecules were examined by western blot analysis. One representative experiment of three is shown. Similar results were obtained in three independent experiments. c IHC scores for DUSP1 staining in the indicated groups. Error bars represent the mean \pm s.d. $(n=24$, Wilcoxon signed rank test). $* * P<0.01$. d Representative IHC images for USP33 and DUSP1 expression in tissue microarrays. e Correlation between the IHC scores for USP33 and DUSP1 $(n=51$, Pearson correlation assay).

\section{Discussion}

Docetaxel was the first cytotoxic therapy associated with a survival benefit in CRPC patients [29]. However, almost all patients will develop docetaxel resistance after treatment [5]. Anti-apoptosis is one of the important mechanisms that decrease the sensitivity of prostate cancer to docetaxel [30]. JNK signalling has been reported to be involved in the regulation of docetaxel-induced apoptosis in triple-negative breast cancer and prostate cancer [31, 4]. Our study suggests that USP33 contributes to docetaxel resistance in prostate cancer cells including the ARnegative PC3 cells, thus illustrating its negative regulatory role in docetaxel-induced apoptosis in prostate cancer, whereby USP33 interacts with and stabilises DUSP1 so that DUSP1 can efficiently dephosphorylate JNK and then inhibit JNK-mediated apoptosis. Our study provides new evidence for understanding the mechanism underlying the resistance to docetaxel-induced apoptosis in prostate cancer. More importantly, this mechanism is still effective in androgen-independent prostate cancer cells. Therefore, our study indicates that interfering 
a

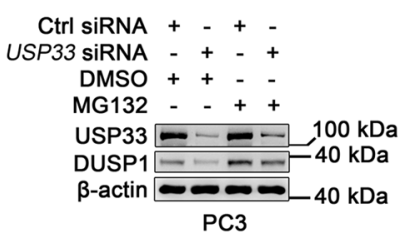

b
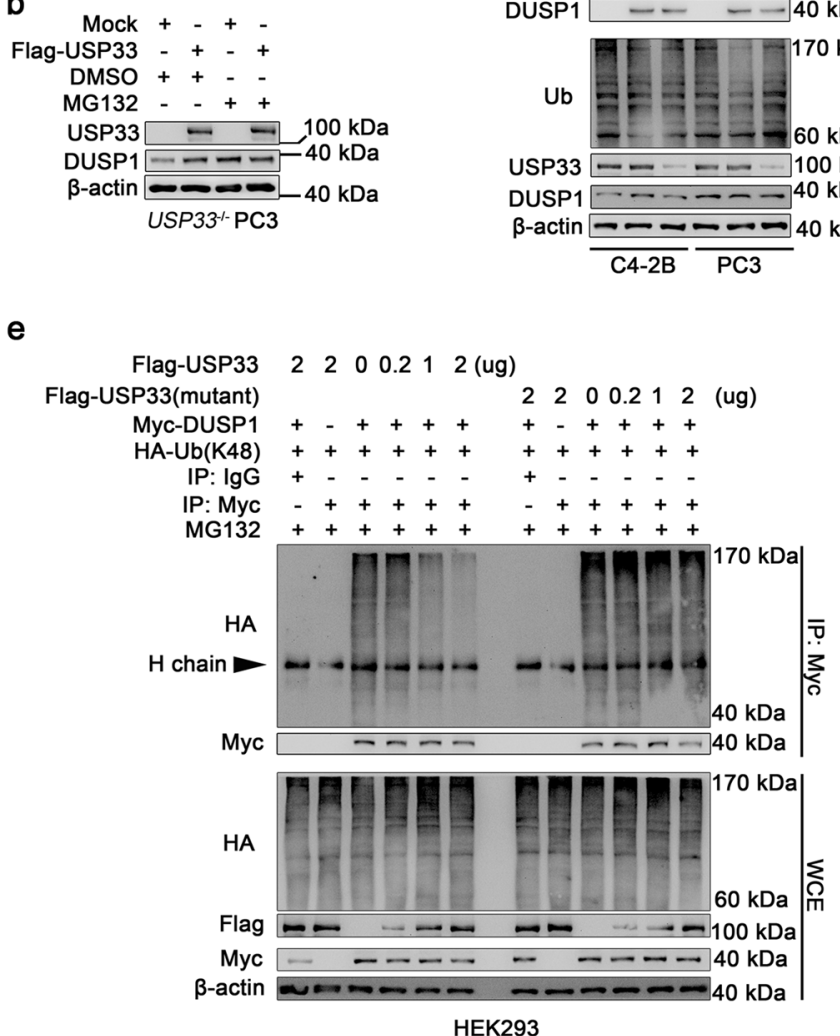

d

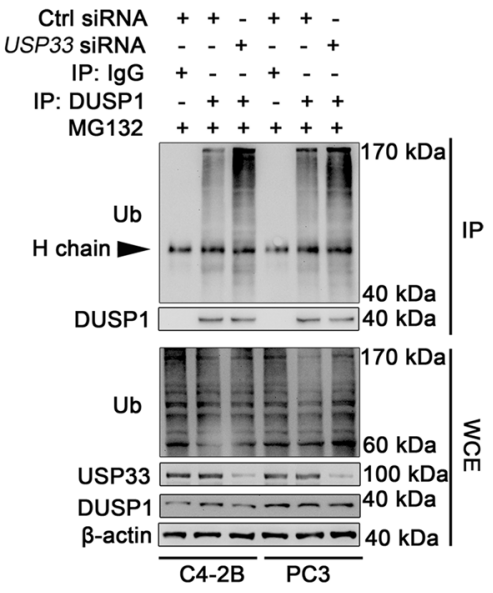

Ctrl siRnA + + + + +

USP33 SiRNA - + + + +

$\mathrm{IP}: \lg \mathrm{G}+\cdots+\cdots$

IP: DUSP1 - + + + +

MG132 +++++

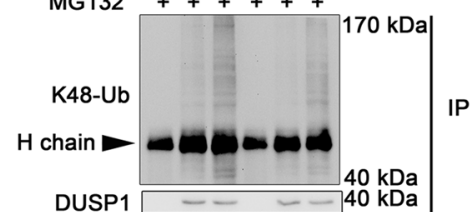

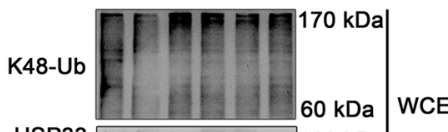

USP33 - - - - $100 \mathrm{kDa}$

DUSP1 $--\cdots--40 \mathrm{kDa}$

$\beta$-actin $-\square-\square-\square 0 \mathrm{kDa}$

C4-2B PC3

f

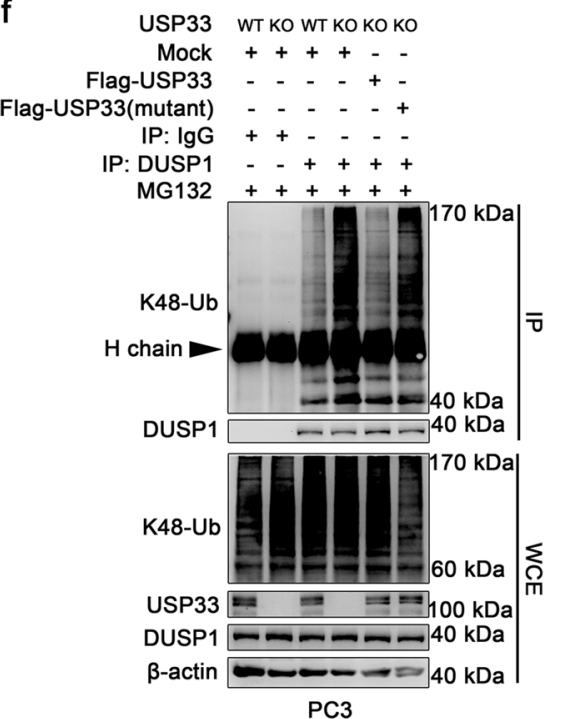

Fig. 6 USP33 stabilises DUSP1 by removing K48-linked polyubiquitin chains from DUSP1. a, b PC3 cells were transfected with Ctrl siRNA or USP33 siRNA (USP33 RNAi \#1) (a), and USP33 ${ }^{-1-}$ PC3 cells were transfected with Mock or Flag-USP33 vector for $48 \mathrm{~h}$ (b) in the presence or absence of MG132 $(10 \mu \mathrm{M})$ as indicated. The indicated molecules were examined by western blot analysis. c C4-2B and PC3 cells were treated with control (Ctrl) or USP33 siRNA (USP33 RNAi \#1) for $48 \mathrm{~h}$ in the presence of MG132 $(10 \mu \mathrm{M})$. Polyubiquitination of DUSP1 was examined by western blot (WB) analysis after immunoprecipitation (IP) with an anti-DUSP1 antibody. WCE, whole-cell extracts. d C4-2B and PC3 cells were transfected with control (Ctrl) or USP33 siRNA (USP33 RNAi \#1) for $48 \mathrm{~h}$ in the presence of MG132 $(10 \mu \mathrm{M})$. The K48 polyubiquitination of DUSP1 was examined by western blot (WB) assay using a K48-Ub specific antibody after immunoprecipitation (IP) with anti-DUSP1 or IgG antibody. e HEK293 cells were cotransfected with the indicated

with USP33 or DUSP1 may be used in the adjuvant treatment of CRPC.

Poly-Ub modification is one of the most important posttranslational modifications in regulating tumours and other diseases [6]. E3 ubiquitin ligases have been found to be closely associated with tumour growth and drug resistance. amounts $(0,0.2,1$ or $2 \mu \mathrm{g})$ of Flag-USP33 or Flag-USP33(mutant) and equal amounts of Myc-DUSP1 and HA-Ub (K48) vectors for $48 \mathrm{~h}$ in the presence of MG132 $(10 \mu \mathrm{M})$. Then, K48-polyubiquitinated DUSP1 was examined by western blot (WB) of HA after immunoprecipitation (IP) with anti-Myc agarose. WCE, whole-cell extracts. f USP33 WT $\left(\mathrm{USP}^{+/+}\right)$and USP33 KO (USP33 $3^{-/-}$) PC3 cells were transfected with Mock, Flag-USP33 or Flag-USP33 (mutant) vector as indicated for $48 \mathrm{~h}$ in the presence of MG132 $(10 \mu \mathrm{M})$. The K48 polyubiquitination of DUSP1 was examined by western blot (WB) with K48-Ub-specific antibody after immunoprecipitation (IP) with antiDUSP1 or IgG antibody. MG132 in all experiments were added $6 \mathrm{~h}$ before cells were harvested. All cell lysates that were tested for ubiquitination in $\mathbf{c}-\mathbf{f}$ were heat denatured in the presence of $1 \%$ SDS before IP. One representative experiment of three is shown. Similar results were obtained in three independent experiments.

In recent years, deubiquitinases have also been gradually revealed to be new targets for cancer therapy [32]. Deubiquitinating enzymes have been implicated in regulating the degradation and activity of substrate proteins by removing poly-Ub chains (e.g., K48 or K63 types) from substrate proteins. A growing number of deubiquitinases have been 

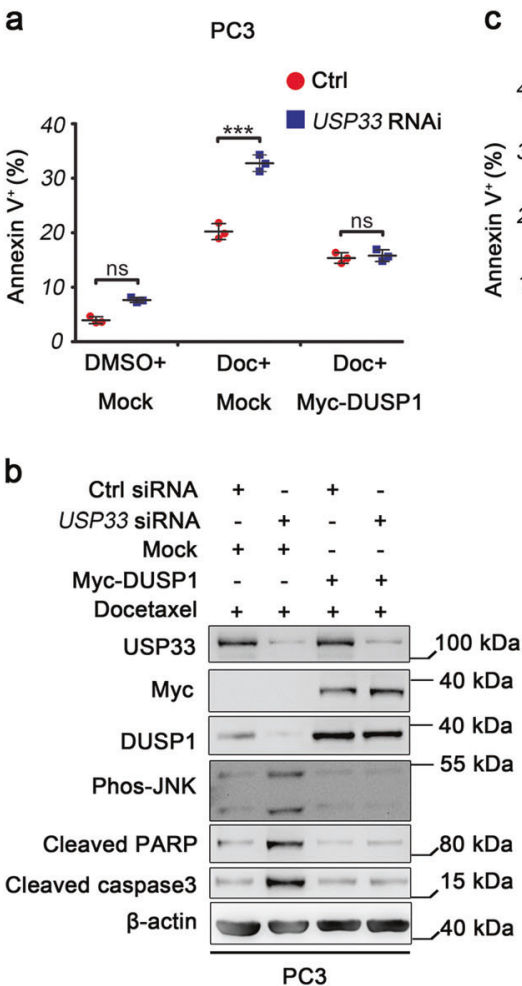

Fig. 7 DUSP1 and USP33 synergistically inhibit docetaxel-induced activation of JNK and apoptosis of PC3 cells. a PC3 cells were cotransfected with control (Ctrl) or USP33 siRNA (USP33 RNAi \#1) and Mock or Myc-DUSP1 vector for $48 \mathrm{~h}$ and then treated with DMSO or docetaxel $(10 \mathrm{nM})$ for $48 \mathrm{~h}$ as indicated. Annexin $\mathrm{V}^{+}$apoptotic cells were quantified by Annexin V/PI assay. b PC3 cells were cotransfected with control (Ctrl) or USP33 siRNA (USP33 RNAi \#1) and Mock or Myc-DUSP1 vector for $48 \mathrm{~h}$ as indicated in the presence of docetaxel $(10 \mathrm{nM})$ for $24 \mathrm{~h}$, and the indicated molecules were examined by western blot analysis. One representative experiment of three is shown. Similar results were obtained in three independent experiments. c USP $33^{+/+}$and USP $33^{-1-}$ PC3 cells were transfected with control (Ctrl) or DUSP1 siRNAs for $48 \mathrm{~h}$ as indicated and then treated

associated with cancer and anticancer therapy directly or indirectly [33, 34]. For example, COP9 signalosome 5 (CSN5) can enable tumour cells to be more sensitive to antiCTLA4 therapy through its ability to deubiquitinate and stabilise PD-L1 [35]. In addition, deubiquitinase DUB3 in colorectal cancer promotes NRF2 stability and transcriptional activity by decreasing the K48-linked ubiquitination of NRF2, which increases chemotherapy resistance in colon cancer cell lines [36]. Deubiquitinase JOSD1 deubiquitinates and stabilises MCL1 to suppress mitochondrial apoptotic signalling and causes chemoresistance in gynaecological cancer [37]. Our study suggests that USP33 is responsible for the stabilisation of DUSP1 by demonstrating that USP33 is required for the removal of K48 polyubiquitin chains from DUSP1, which then inhibits DUSP1 degradation by the ubiquitin-proteasome pathway. However, why USP33 is upregulated in AR-negative prostate cancer cells and how the function of USP33 is regulated still await further investigations.

DUSP1 is a threonine/tyrosine dual-specificity phosphatase that mainly mediates the dephosphorylation of ERK1/2, JNK and p38 MAPK by directly dephosphorylating threonine and tyrosine residues [18]. DUSP1 is overexpressed in many cancer types, such as breast, lung, prostate, ovarian, pancreatic and gastric adenocarcinoma [38]. In breast cancer, non-small-cell lung cancer and ovarian cancer, DUSP1 protects cancer cells from chemotherapy-induced apoptosis by dephosphorylating JNK and p38 MAPK [20, 39, 40]. However, DUSP1deficient mice are more susceptible to head and neck squamous cell carcinoma as a result of elevated IL- $1 \beta$ production and an inflammatory microenvironment through enhanced p38 MAPK activation in macrophages but not tumour cells [41]. In human hepatocellular carcinoma 
a

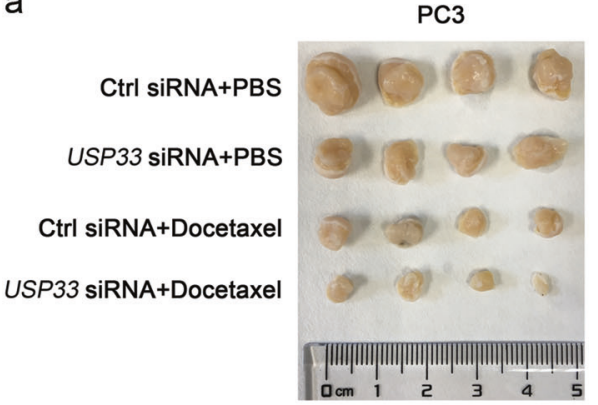

C

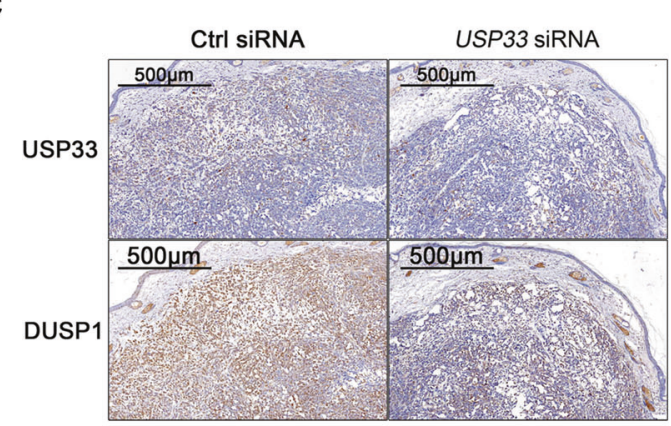

e

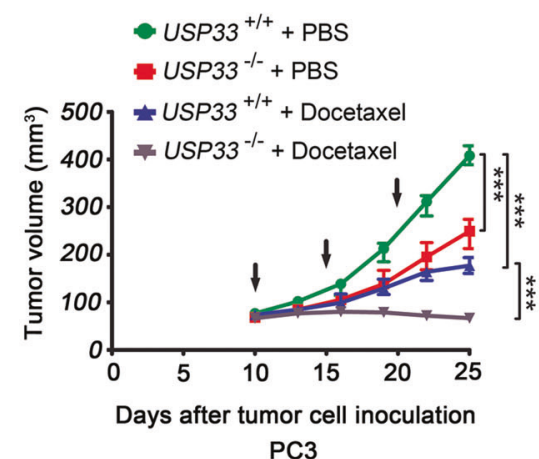

PC3

Fig. 8 USP33 knockdown or knockout inhibits tumour growth and increases the sensitivity of prostate cancer cells to docetaxel. a, b Tumour volume was measured 3 days after the indicated treatments. When the PC3 tumour volume reached $70-100 \mathrm{~mm}^{3}$ (day 7), the tumours with the required size $(n=4)$ were treated as indicated. Black arrows indicate the siRNA injections. Docetaxel was intraperitoneally injected at $10 \mathrm{mg} / \mathrm{kg}$ once every 5 days from the time the tumour reached the required size $\left(70-100 \mathrm{~mm}^{3}\right)$ for a total of three times. c Representative IHC images of USP33 and DUSP1 protein staining in tumour tissues. d, e Tumour volume was measured 5 days after the

(HCC), DUSP1 is degraded by the SKP2/CKS1 ubiquitin ligase complex via the ubiquitin-proteasome pathway, which may further promote HCC growth by maintaining ERK1/2 activation [42]. Thus, DUSP1 demonstrates different regulatory modes in different cells. Importantly, the regulation of DUSP1 degradation and the function of DUSP1 in prostate cancer remain largely unknown. Our study revealed that K48 polyubiquitination of DUSP1 can be reversed by USP33 in prostate cancer, leading to high expression of DUSP1, low activity of JNK1/2 and apoptosis resistance during docetaxel treatment of prostate cancer and
PC3

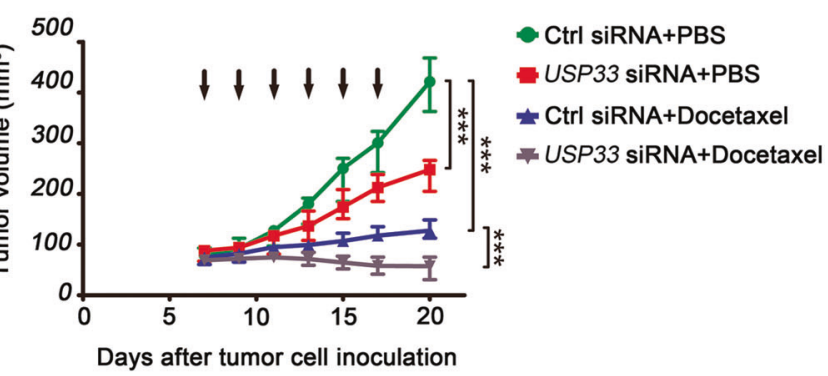

d

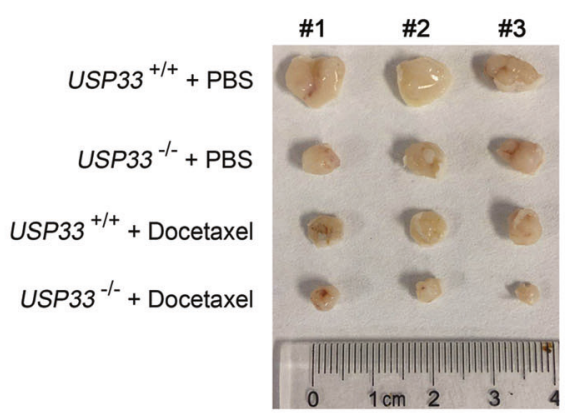

f

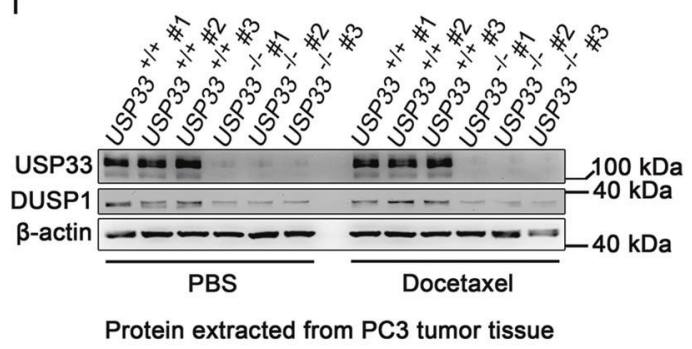

indicated treatments. When USP $33^{+/+}$and USP $33^{-1-}$ PC3 tumour volumes reached $70-100 \mathrm{~mm}^{3}$ (day 10), the tumours with required sizes $(n=3)$ were treated as indicated. Docetaxel was intraperitoneally injected at $10 \mathrm{mg} / \mathrm{kg}$ once every 5 days for a total of three times. Black arrows indicate the docetaxel injections. Error bars in $\mathbf{b}$ and $\mathbf{e}$ represent the mean \pm s.d. $* * * P<0.001$ (one-way ANOVA followed by Bonferroni multiple comparison using PRISM software). f Protein was extracted from each tumour tissue, and the indicated molecules were examined by western blot analysis. \#1-\#3 is the number of the tumours in $\mathbf{d}$.

USP33-DUSP1-JNK is a new regulatory pathway in regulating docetaxel resistance.

In summary, our study has convincingly demonstrated that USP33 plays an important role in docetaxel-induced apoptosis of prostate cancer by removing the K48 polyubiquitin chain from DUSP1 to stabilise DUSP1 and ultimately inhibit JNK-mediated apoptosis in prostate cancer cells including androgen-independent prostate cancer cells. Our data suggest that USP33 and DUSP1 may be potential new targets for combination chemotherapy in castrationresistant prostate cancer. 
Acknowledgements We thank Ms. Meng Qiao and Dr. Chenguang Bai for technical assistance. This work was supported by the Shanghai Clinical Medical Center of Urologic Diseases Program (2017ZZ01005), the Research fund of Shanghai municipal health and family planning commission (2017Y0019), and the Natural Science Foundation of Shanghai (18ZR1438400).

\section{Compliance with ethical standards}

Conflict of interest The authors declare that they have no conflict of interest.

Publisher's note Springer Nature remains neutral with regard to jurisdictional claims in published maps and institutional affiliations.

\section{References}

1. Siegel RL, Miller KD, Jemal A. Cancer statistics, 2018. CA Cancer J Clin. 2018;68:7-30.

2. Chen W, Zheng R, Baade PD, Zhang S, Zeng H, Bray F, et al. Cancer statistics in China, 2015. CA Cancer J Clin. 2016;66:115-32.

3. Galsky MD, Small AC, Tsao CK, Oh WK. Clinical development of novel therapeutics for castration-resistant prostate cancer: historic challenges and recent successes. CA Cancer J Clin. 2012;62: 299-308.

4. Liu Q, Harvey CT, Geng H, Xue C, Chen V, Beer TM, et al. Malate dehydrogenase 2 confers docetaxel resistance via regulations of JNK signaling and oxidative metabolism. Prostate. 2013;73:1028-37.

5. Karanika S, Karantanos T, Kurosaka S, Wang J, Hirayama T, Yang G, et al. GLIPR1- $\Delta$ TM synergizes with docetaxel in cell death and suppresses resistance to docetaxel in prostate cancer cells. Mol Cancer. 2015;14:122.

6. Senft D, Qi J, Ronai ZA. Ubiquitin ligases in oncogenic transformation and cancer therapy. Nat Rev Cancer. 2018;18:69-88.

7. Mevissen TET, Komander D. Mechanisms of deubiquitinase specificity and regulation. Annu Rev Biochem. 2017;86:159-92.

8. Fraile JM, Quesada V, Rodríguez D, Freije JM, López-Otín C. Deubiquitinases in cancer: new functions and therapeutic options. Oncogene. 2012;31:2373-88.

9. Pal A, Young MA, Donato NJ. Emerging potential of therapeutic targeting of ubiquitin-specific proteases in the treatment of cancer. Cancer Res. 2014;74:4955-66.

10. Li Z, Na X, Wang D, Schoen SR, Messing EM, Wu G. Ubiquitination of a novel deubiquitinating enzyme requires direct binding to von Hippel-Lindau tumor suppressor protein. J Biol Chem. 2002;277:4656-62.

11. Li J, D'Angiolella V, Seeley ES, Kim S, Kobayashi T, Fu W, et al. USP33 regulates centrosome biogenesis via deubiquitination of the centriolar protein CP110. Nature. 2013;495:255-9.

12. Yuasa-Kawada J, Kinoshita-Kawada M, Rao Y, Wu JY. Deubiquitinating enzyme USP33/VDU1 is required for Slit signaling in inhibiting breast cancer cell migration. Proc Natl Acad Sci USA. 2009;106:14530-5.

13. Liu H, Zhang Q, Li K, Gong Z, Liu Z, Xu Y, et al. Prognostic significance of USP33 in advanced colorectal cancer patients: new insights into $\beta$-arrestin-dependent ERK signaling. Oncotarget. 2016;7:81223-40.

14. Huang Z, Wen P, Kong R, Cheng H, Zhang B, Quan C, et al. USP33 mediates Slit-Robo signaling in inhibiting colorectal cancer cell migration. Int J Cancer. 2015;136:1792-802.

15. Wen P, Kong R, Liu J, Zhu L, Chen X, Li X, et al. USP33, a new player in lung cancer, mediates Slit-Robo signaling. Protein Cell. 2014;5:704-13.
16. Tammy LR, Gang W, Holt RobertA, Jones StevenJM, Marra MarcoA, Sadar. MarianneD. Identification of novel androgenresponsive genes by sequencing of LongSAGE libraries. BMC Genomics. 2009;10:476.

17. Yuting S, Bu-Er W, Kevin GL, Peng Y, Li L, Suchit J, et al. Androgen deprivation causes epithelial-mesenchymal transition in the prostate: implications for androgen-deprivation therapy. Cancer Res. 2011;72:527-36.

18. Keyse SM. Dual-specificity MAP kinase phosphatases (MKPs) and cancer. Cancer Metastasis Rev. 2008;27:253-61.

19. Magi-Galluzzi C, Mishra R, Fiorentino M, Montironi R, Yao H, Capodieci P, et al. Mitogen-activated protein kinase phosphatase 1 is overexpressed in prostate cancers and is inversely related to apoptosis. Lab Invest. 1997;76:37-51.

20. Chattopadhyay S, Machado-Pinilla R, Manguan-García C, BeldaIniesta C, Moratilla C, Cejas P, et al. MKP1/CL100 controls tumor growth and sensitivity to cisplatin in non-small-cell lung cancer. Oncogene. 2006;25:3335-45.

21. Liu F, Gore AJ, Wilson JL, Korc M. DUSP1 is a novel target for enhancing pancreatic cancer cell sensitivity to gemcitabine. PLoS ONE. 2014;9:e84982.

22. Small GW, Shi YY, Higgins LS, Orlowski RZ. Mitogen-activated protein kinase phosphatase-1 is a mediator of breast cancer chemoresistance. Cancer Res. 2007;67:4459-66.

23. Wang C, Chen T, Zhang J, Yang M, Li N, Xu X, et al. The E3 ubiquitin ligase Nrdp1 'preferentially' promotes TLR-mediated production of type I interferon. Nat Immunol. 2009;10:744-52.

24. Chen T, Yang M, Yu Z, Tang S, Wang C, Zhu X, et al. Small GTPase RBJ mediates nuclear entrapment of MEK1/MEK2 in tumor progression. Cancer Cell. 2014;25:682-96.

25. Yang M, Chen T, Li X, Yu Z, Tang S, Wang C, et al. K33-linked polyubiquitination of Zap70 by Nrdp1 controls CD8(+) T cell activation. Nat Immunol. 2015;16:1253-62.

26. Cornford P, Bellmunt J, Bolla M, Briers E, De Santis M, Gross T, et al. EAU-ESTRO-SIOG guidelines on prostate cancer. Part II: treatment of relapsing, metastatic, and castration-resistant prostate cancer. Eur Urol. 2017;71:630-42.

27. Cookson MS, Roth BJ, Dahm P, Engstrom C, Freedland SJ, Hussain M, et al. Castration-resistant prostate cancer: AUA Guideline. J Urol. 2013;190:429-38.

28. Berthouze M, Venkataramanan V, Li Y, Shenoy SK. The deubiquitinases USP33 and USP20 coordinate beta2 adrenergic receptor recycling and resensitization. EMBO J. 2009;28:1684-96.

29. Clara Hwang. Overcoming docetaxel resistance in prostate cancer: a perspective review. Ther Adv Med Oncol. 2012;4:329-40.

30. Ganju A, Yallapu MM, Khan S, Behrman SW, Chauhan SC, Jaggi $\mathrm{M}$. Nanoways to overcome docetaxel resistance in prostate cancer. Drug Resist Updat. 2014;17:13-23.

31. Dávila-González D, Choi DS, Rosato RR, Granados-Principal SM, Kuhn JG, Li WF, et al. Pharmacological inhibition of NOS activates ASK1/JNK pathway augmenting docetaxel-mediated apoptosis in triple-negative breast cancer. Clin Cancer Res. 2018;24:1152-62.

32. Hoeller D, Dikic I. Targeting the ubiquitin system in cancer therapy. Nature. 2009;458:438-44.

33. Amerik AY, Hochstrasser M. Mechanism and function of deubiquitinating enzymes. Biochim Biophys Acta. 2004;1695: 189-207.

34. Wei R, Liu X, Yu W, Yang T, Cai W, Liu J, et al. Deubiquitinases in cancer. Oncotarget. 2015;6:12872-89.

35. Lim SO, Li CW, Xia W, Cha JH, Chan LC, Wu Y, et al. Deubiquitination and stabilization of PD-L1 by CSN5. Cancer Cell. 2016;30:925-39.

36. Zhang Q, Zhang ZY, Du H, Li SZ, Tu R, Jia YF, et al. DUB3 deubiquitinates and stabilizes NRF2 in chemotherapy resistance of colorectal cancer. Cell Death Differ. 2019;26:2300-13. 
37. Wu X, Luo Q, Zhao P, Chang W, Wang Y, Shu T, et al. JOSD1 inhibits mitochondrial apoptotic signalling to drive acquired chemoresistance in gynaecological cancer by stabilizing MCL1. Cell Death Differ. 2019. https://doi.org/10.1038/ s41418-019-0339-0.

38. Shen J, Zhang Y, Yu H, Shen B, Liang Y, Jin R, et al. Role of DUSP1/MKP1 in tumorigenesis, tumor progression and therapy. Cancer Med. 2016;5:2061-8.

39. Wang HY, Cheng Z, Malbon CC. Overexpression of mitogenactivated protein kinase phosphatases MKP1, MKP2 in human breast cancer. Cancer Lett. 2003;191:229-37.
40. Kang Y, Nagaraja AS, Armaiz-Pena GN, Dorniak PL, Hu W, Rupaimoole R, et al. Adrenergic stimulation of DUSP1 impairs chemotherapy response in ovarian cancer. Clin Cancer Res. 2016;22:1713-24.

41. Zhang X, Hyer JM, Yu H, D'Silva NJ, Kirkwood KL. DUSP1 phosphatase regulates the proinflammatory milieu in head and neck squamous cell carcinoma. Cancer Res. 2014;74:7191-7.

42. Calvisi DF, Pinna F, Meloni F, Ladu S, Pellegrino R, Sini M, et al. Dual-specificity phosphatase 1 ubiquitination in extracellular signal-regulated kinase-mediated control of growth in human hepatocellular carcinoma. Cancer Res. 2008;68:4192-4200. 